Original article

\title{
Polyphenol intake and cardiovascular risk factors in a population with type 2 diabetes: The TOSCA.IT study
}

Marilena Vitale ${ }^{a}$, Olga Vaccaro ${ }^{a}$, Maria Masulli a , Enzo Bonora ${ }^{b}$, Stefano Del Prato ${ }^{\text {, }}$

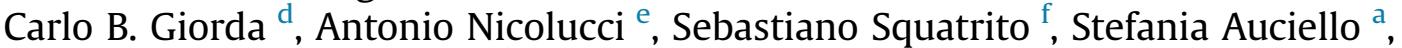
Anna C. Babini ${ }^{\mathrm{g}}$, Laura Bani $^{\mathrm{h}}$, Raffaella Buzzetti ${ }^{\mathrm{i}}$, Emanuela Cannarsa ${ }^{\mathrm{j}}$, Mauro Cignarelli ${ }^{\mathrm{k}}$, Massimo Cigolini ${ }^{\mathrm{b}}$, Gennaro Clemente ${ }^{1}$, Sara Cocozza ${ }^{\mathrm{a}}$, Laura Corsi ${ }^{\mathrm{m}}$, Federica D'Angelo ${ }^{n}$, Elisabetta Dall'Aglio ${ }^{\circ}$, Graziano Di Cianni ${ }^{\mathrm{p}}$, Lucia Fontana ${ }^{\mathrm{q}}$, Giovanna Gregori ${ }^{\mathrm{r}}$, Sara Grioni ${ }^{\mathrm{s}}$, Carla Giordano ${ }^{\mathrm{t}}$, Rossella Iannarelli ${ }^{\mathrm{u}}$, Ciro Iovine ${ }^{\mathrm{a}}$, Annunziata Lapolla ${ }^{v}$, Davide Lauro ${ }^{\mathrm{w}}$, Luigi Laviola ${ }^{\mathrm{x}}$, Chiara Mazzucchelli ${ }^{\mathrm{y}}$, Stefano Signorini ${ }^{\mathrm{h}}$, Laura Tonutti ${ }^{\mathrm{z}}$, Roberto Trevisan ${ }^{\text {aa }}$, Chiara Zamboni ${ }^{\mathrm{ab}}$, Gabriele Riccardi a, Angela A. Rivellese ${ }^{\text {a, }}$, on behalf of the TOSCA.IT Study Group

a Dipartimento di Medicina Clinica e Chirurgia, Università "Federico II", Naples, Italy

b Dipartimento di Endocrinologia, Diabetologia e Metabolismo, Università di Verona, Italy

${ }^{\mathrm{c}}$ Dipartimento di Medicina Clinica e Sperimentale, Università di Pisa, Italy

d ASL Torino 5, Chieri, Italy

e CORESEARCH, Center for Outcomes Research and Clinical Epidemiology, Pescara, Italy

f Dipartimento di Medicina Clinica e Sperimentale, Università di Catania, Italy

${ }^{g}$ Diabetologia, Ospedale Infermi di Rimini, Italy

${ }^{\mathrm{h}}$ Dipartimento di Patologia Sperimentale, Ospedale di Desio, Università di Milano Bicocca, Italy

${ }^{\text {i } U O C ~ d i ~ D i a b e t o l o g i a ~ U n i v e r s i t a r i a, ~ O s p e d a l e ~ S a n t a ~ M a r i a ~ G o r e t t i, ~ L a t i n a, ~ I t a l y ~}$

${ }^{\mathrm{j}}$ UOSD Diabetologia, Presidio Ospedaliero, Atri, Italy

${ }^{\mathrm{k}}$ UO Endocrinologia e Malattie del Metabolismo, AOU di Foggia, Italy

${ }^{1}$ IRPPS Research National Council, Penta di Fisciano, Salerno, Italy

m SSD Diabetologia e Malattie del Metabolismo, ASL 4 Chiavarese, Genova, Italy

${ }^{n}$ UOC Malattie Metaboliche e Diabetologia, INRCA-IRCCS Institute, Ancona, Italy

${ }^{\circ}$ Dipartimento di Medicina Clinica e Sperimentale, Università di Parma, Italy

p UOC Diabetologia, ASL 6, Livorno, Italy

${ }^{\mathrm{q}}$ UOC Diabetologia e Dietologia, Ospedale Pertini, Roma, Italy

${ }^{\mathrm{r}}$ UO Diabetologia e Malattie del Metabolismo, USL1 di Massa e Carrara, Italy

${ }^{s}$ Unità di Epidemiologia e Prevenzione, Fondazione IRCCS, Istituto Nazionale Tumori, Milano, Italy

${ }^{\mathrm{t}}$ Dipartimento di Endocrinologia e Malattie Metaboliche, Università di Palermo, Italy

u UOSD Diabetologia e Malattie del Metabolismo, Ospedale San Salvatore, L'Aquila, Italy

v Dipartimento di Medicina, Università di Padova, Italy

${ }^{\mathrm{w}}$ Dipartimento di Medicina Interna, Università di Tor Vergata, Roma, Italy

x Dipartimento Emergenza e Trapianti di Organi, Università “Aldo Moro”, Bari, Italy

y DIMI, Università di Genova, IRCCS San Martino, Italy

${ }^{z}$ SOC di Endocrinologia e Malattie del Metabolismo, AOU S. Maria della Misericordia, Udine, Italy

aa USC Malattie Endocrine-Diabetologia, ASST "Papa Giovanni XXIII", Bergamo, Italy

${ }^{\text {ab }}$ UO Malattie Metaboliche, Dietologia e Nutrizione Clinica, AOU Arcispedale S. Anna, Ferrara, Italy

\section{A R T I C L E I N F O}

Article history:

Received 15 July 2016

Accepted 1 November 2016

\section{S U M M A R Y}

Background: The role of polyphenol intake on cardiovascular risk factors is little explored, particularly in people with diabetes. Aim: To evaluate the association between the intake of total polyphenols and polyphenol classes with the major cardiovascular risk factors in a population with type 2 diabetes.

Abbreviations used: TOSCA.IT, Thiazolidinediones or Sulfonylureas and Cardiovascular Accidents Intervention Trial; BMI, body mass index; CRP, C-reactive protein; HbA1c,

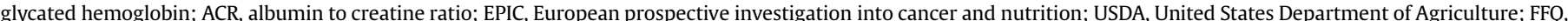

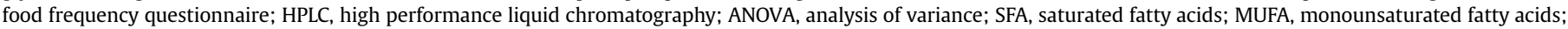
PUFA, polyunsaturated fatty acids.

* Corresponding author. Dept. of Clinical Medicine and Surgery, “Federico II" University of Naples, Via S. Pansini, n.5, 80131 Naples, Italy. Fax: +39 0815466152.

E-mail address: rivelles@unina.it (A.A. Rivellese). 
Keywords:

Dietary polyphenols

Flavonoids

Phenolic acids

Cardiovascular risk

Type 2 diabetes
Methods: Dietary habits were investigated in 2573 males and females participants of the TOSCA.IT study. The European Prospective Investigation on Cancer and Nutrition (EPIC) questionnaire was used to assess dietary habits. In all participants, among others, we assessed anthropometry, plasma lipids, blood pressure, C-reactive protein and HbA1c following a standard protocol. The USDA and Phenol-Explorer databases were used to estimate the polyphenol content of the habitual diet.

Results: Average intake of polyphenols was $683.3 \pm 5.8 \mathrm{mg} / \mathrm{day}$. Flavonoids and phenolic acids were the predominant classes (47.5\% and 47.4\%, respectively). After adjusting for potential confounders, people with the highest intake of energy-adjusted polyphenols (upper tertile) had a more favorable cardiovascular risk factors profile as compared to people with the lowest intake (lower tertile) (BMI was 30.7 vs $29.9 \mathrm{~kg} / \mathrm{m}^{2}$, HDL-cholesterol was $45.1 \mathrm{vs} 46.9 \mathrm{mg} / \mathrm{dl}$, LDL-cholesterol was $103.2 \mathrm{vs} 102.1 \mathrm{mg} / \mathrm{dl}$, triglycerides were 153.4 vs $148.0 \mathrm{mg} / \mathrm{dl}$, systolic and diastolic blood pressure were respectively 135.3 vs 134.3 and 80.5 vs $79.6 \mathrm{~mm} / \mathrm{Hg}$, HbA1c was 7.70 vs $7.67 \%$, and C-reactive Protein was 1.29 vs $1.25 \mathrm{mg} / \mathrm{dl}$, $\mathrm{p}<.001$ for all). The findings were very similar when the analysis was conducted separately for flavonoids or phenolic acids, the two main classes of polyphenols consumed in this population.

Conclusions: Polyphenol intake is associated with a more favorable cardiovascular risk factors profile, independent of major confounders. These findings support the consumption of foods and beverages rich in different classes of polyphenols particularly in people with diabetes.

Clinical trial: http://www.clinicaltrials.gov; Study ID number: NCT00700856.

(C) 2016 Elsevier Ltd and European Society for Clinical Nutrition and Metabolism. All rights reserved.

\section{Introduction}

In the last few years, the interest for polyphenols in the field of human nutrition has rapidly increased for their possible beneficial effects against the development of several chronic diseases - in particular cardiovascular diseases and diabetes [1-4] -, and in improving cardiovascular risk factors [5-9].

Polyphenols are natural bioactive compounds mainly found in plant-based foods, such as fruit, vegetables, legumes, cereals and cocoa, and plant-derived beverages, such as coffee, tea and wine. Based on the biochemical structure, five main classes of polyphenols with different properties and bioavailability have been identified: flavonoids (the group of polyphenols mostly studied), phenolic acids, stilbenes, lignans and others polyphenols [10].

To date, cross-sectional and prospective epidemiological studies have shown that the intake of total polyphenols and/or of specific foods rich in polyphenols, such as tea, coffee, and chocolate, are associated with a better cardiovascular risk factors profile (plasma lipids, glucose, blood pressure, platelet function, biomarkers of inflammation) [11,12] and, in some cases, with a decrease in cardiovascular diseases and diabetes incidence [13].

However, the majority of these studies have been performed in the general population and focused on the intake of flavonoids and their subclasses; indeed data on other types of polyphenols are scant, although they may be equally important, as suggested by some results from the PREDIMED study. In fact, this study showed that a high polyphenol intake, deriving especially from stilbenes and lignans, is associated with lower risk of overall mortality [14], whereas a high intake of total flavonoids (specifically flavanones and dihydroflavonols) and stilbenes is associated with a reduced risk of diabetes [15].

In addition to epidemiological studies, in vitro and animal data and some intervention studies in humans have confirmed and reinforced the evidence for beneficial effects on cardiovascular risk factors mainly lipid and glucose metabolism - of different polyphenols and diets rich in polyphenols from different sources [16-21].

Considering the possible role of polyphenols in ameliorating several cardiovascular risk factors, it is relevant to evaluate the intake of polyphenols and the relation of the different classes of polyphenols with the cardiovascular risk factors profile in people with type 2 diabetes, a population characterized by a high cardiovascular risk.

Therefore, the aim of our study was to evaluate the association between total polyphenol intake and polyphenol classes with the major cardiovascular risk factors (glucose control, lipid profile, blood pressure) and biomarkers of inflammation in a large population of people with type 2 diabetes. To the best of our knowledge, this is the first epidemiological observational study evaluating these aspects in type 2 diabetic patients.

\section{Materials and methods}

\subsection{Study population}

This cross-sectional study was conducted in 2573 people with type 2 diabetes, diagnosed according to ADA criteria [22], 1535 men and 1038 women, aged 50-75 years, enrolled in the TOSCA.IT study, a randomized clinical trial designed to compare the impact of glucose lowering drugs on cardiovascular events (http://www. clinicaltrials.gov, study ID number: NCT00700856). The study protocol has been already published [23]. The study participants were recruited in 60 centers distributed all over Italy. The study protocol was approved by the Ethics Review Committee of the Coordinating Center and of each participating center, and written informed consent was obtained from all participants before entering the study. For the purposes of the present analysis, baseline data collected prior to randomization to study treatment - were used. Among the other parameters evaluated, body weight, height, waist and hip circumference, blood pressure, were measured according to standard procedures. Body Mass Index (BMI) was calculated as weight $(\mathrm{kg}) /$ height $\left(\mathrm{m}^{2}\right)$. Blood samples were obtained in the morning after an overnight fast. All biochemical analyses were performed in a central laboratory. Total cholesterol, HDLcholesterol, triglycerides and C-reactive protein (PCR) were measured by standard methods. LDL-cholesterol was calculated according to the Friedewald equation only for triglyceride values $<400 \mathrm{mg} / \mathrm{dl}$. Glycated hemoglobin (HbA1c) was measured with High Liquid Performance Chromatography. At baseline, all participants were treated with metformin, as per study inclusions criteria. Data on the use of other medications were also collected.

\subsection{Dietary assessment}

Dietary habits were assessed with the Italian version of the European Prospective Investigation into Cancer and Nutrition (EPIC) questionnaire [24]. The questionnaire contains 248 items including the type of fat used as seasoning, or added after cooking. The person 
filling in the questionnaire indicates the absolute frequency of consumption of each item (per day, week, month or year) and the quantity of the food consumed, selecting the image of a food portion showing a small, medium and large portion size, with additional quantifiers (e.g. "smaller than the small portion" or "between the small and medium portion" etc ...). The nutrient composition of the diet was calculated with a software containing the Italian Food Tables. Eighty-six incomplete questionnaires and questionnaires with implausible data (i.e. energy intake less than 800 or greater than $5000 \mathrm{Kcal} /$ day) were excluded from the analyses.

\subsection{Estimation of polyphenol intake}

The intake of polyphenols and their classes was evaluated using the USDA database [25] in combination with the Phenol-Explorer ${ }^{\circledR}$ database [26] to enable the examination of the polyphenol content of as many foods as possible. Missing data from cereal products, such as pizza, polenta, biscuits, crisp toasts, crackers and breadsticks, were estimated based on refined wheat flour data from Phenol-Explorer ${ }^{\circledR}$ database. For processed foods such as canned foods and pre-packaged meals, the estimation was made according to the percentages of ingredients in the food products. Complex foods such as cakes and sauces were calculated as recipes; the individual ingredients were obtained from data available on the Internet. Different types of coffee, "espresso" and "decaffeinated", were reported in the food frequency questionnaire. The polyphenol content of "espresso" coffee was estimated on the coffee brand frequently used in Italy; therefore, the mean polyphenol content in the Arabica and Robusta varieties from the Phenol-Explorer database was calculated and multiplied by 2 to avoid underestimation of polyphenol from coffee espresso, as suggested by Parras [27]. For "decaffeinated" coffee, the polyphenol content of "decaffeinated filtered coffee" from the Phenol-Explorer database was considered.

The polyphenol composition of tea was obtained from a mix of herbal teas considering the unspecified type of herbal tea in the food frequency questionnaire (FFQ).

Finally, individual polyphenol intake from each food was calculated by multiplying the content of each polyphenol by the daily consumption of each food. Mean intake of each polyphenol class (flavonoids, phenolic acids, stilbenes, lignans and other polyphenols) was determined. Total polyphenol intake was calculated as the sum of all individual polyphenol intakes from all the food sources reported by the FFQ.

The cut-off used for foods to be included in the polyphenol estimation was based on a previous study which referred to foods that contributed $<1 \mathrm{mg} /$ day to the overall polyphenol intake as minor contributors. Thus, foods containing less than $1 \mathrm{mg}$ of polyphenols as consumed in a usual portion were excluded from the analysis.

\subsection{Statistical analysis}

Data from continuous variables are presented as mean \pm standard deviation. Percentages are used for categorical variables. Not normally distributed variables were logarithmically transformed for statistical analyses and back-transformed to natural units for presentation in the text and tables.

Polyphenol intake is reported as $\mathrm{mg}$ of polyphenols per $1000 \mathrm{kcal}$ to correct for total energy intake, which was higher in subjects with increased polyphenol consumption. Baseline characteristics and metabolic profile have been evaluated across tertiles of total polyphenol intake and of the two main polyphenol classes (flavonoids and phenolic acids). Differences between tertiles were tested by a one-factor analysis of variance (ANOVA) and Post-Hoc test for multiple comparisons (Bonferroni Test) for continuous variables, and by the chi-square test for categorical variables. The analysis was adjusted for gender, age, and all the variables which were significantly different between tertiles, i.e. BMI, waist and hip circumferences, smoke, alcohol intake, dietary lipids, saturated SFA -, monounsaturated - MUFA -, and polyunsaturated - PUFAfatty acids, added sugars and fiber intake.

All P-values reported were based on two-sided tests, the significance level was set at .05. IBM SPSS Statistic 20 (SPSS Inc., Chicago, IL, USA) software was used for all the statistical analysis.

\section{Results}

The total polyphenol intake of our population was $683.3 \pm 5.8 \mathrm{mg} /$ day and, adjusting for energy intake, it was $378.7 \pm 4.1 \mathrm{mg} / 1000 \mathrm{Kcal}$ for men and $374.0 \pm 4.9 \mathrm{mg} / 1000 \mathrm{Kcal}$ for women, with no difference between genders. Flavonoids and phenolic acids represented the two main classes of polyphenol intake in our population, accounting for $95 \%$ of the total polyphenol intake (respectively $47.5 \%$ and $47.4 \%$ ); the remaining $5.1 \%$ was represented by the others three classes (other polyphenols, stilbenes, and lignans, respectively $3.8 \%, .8 \%$, and .5\%) (Table 1 ).

Dividing the population according to tertiles of energy-adjusted polyphenol intake, significant differences in some demographic characteristics and in the nutrient composition of the diets were observed (Table 2). In fact, participants with a higher polyphenol intake were older, had lower BMI, waist and hip circumferences, were more likely to be smokers and consumed more alcoholic beverages (mostly wine and beer) ( $\mathrm{p}<.05$ for all). In addition, a higher intake of polyphenols was associated, at least to some extent, to better diet quality. In fact, a lower intake of saturated fatty acids and a higher intake of unsaturated fatty acids and fiber were associated with a higher consumption of polyphenols ( $p<.001$ for all). The groups did not differ in terms of gender distribution and use of lipid and pressure lowering drugs.

Figure 1 shows the metabolic profile, blood pressure values and CRP according to the tertiles of energy-adjusted polyphenol intake. After adjusting for the potential confounders (gender, age, BMI, waist and hip circumferences, smoking, and all nutrients resulted different across the tertiles of energy-adjusted polyphenol intake), people in the higher tertile of energy-adjusted polyphenol intake (tertile 3) showed significantly higher HDL-cholesterol and lower LDL-cholesterol, triglycerides, systolic and diastolic blood pressure, HbA1c, and CRP compared to subjects in the lower tertiles (tertiles 1 and 2$)(p<.001$ for all, analysis of variance and post-hoc test for

Table 1

Percentage contribution of different classes to the total polyphenol intake and main food sources for each class.

\begin{tabular}{|c|c|c|}
\hline & Contribution (\%) & Main food sources in the cohort (\% of total intake within each polyphenol class) \\
\hline Flavonoids & 47.5 & Fruit (32.2\%), Alcoholic beverages ${ }^{\mathrm{a}}$ (22.8\%), Non-alcoholic beverages ${ }^{\mathrm{b}}$ (17.6\%), Vegetables (15.1\%), Legumes (7.5\%) \\
\hline Phenolic acids & 47.4 & 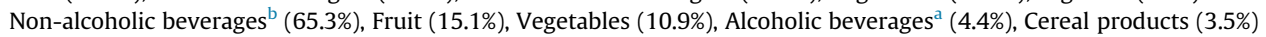 \\
\hline Stilbenes & .8 & Alcoholic beverages $^{\mathrm{a}}(97.1 \%)$ \\
\hline Lignans & .5 & Fruit (85.4\%), Vegetables (6.4\%), Fat and oils (6.3\%) \\
\hline Other Polyphenols & 3.8 & Fat and oils (52.2\%), Cereal products (28.5\%), Alcoholic beverages ${ }^{\mathrm{a}}(13.0 \%)$, Non-alcoholic beverages ${ }^{\mathrm{b}}(6.3 \%)$ \\
\hline
\end{tabular}

a Alcoholic Beverages: wine and beer.

b Non-alcoholic beverages: coffee and tea. 
Table 2

Baseline characteristics and nutrient composition of the diet according to energy-adjusted tertiles of polyphenol intake.

\begin{tabular}{|c|c|c|c|c|}
\hline & Tertile 1 (n 856) & Tertile 2 (n 860) & Tertile 3 (n 858) & P for Anova or $\chi^{2}$ \\
\hline Total polyphenols (mg/1000 kcal) & $\begin{array}{l}228.5 \pm 50.8 \\
(51.8-297.7)\end{array}$ & $\begin{array}{l}355.0 \pm 32.8 \\
(297.9-413.7)\end{array}$ & $\begin{array}{l}546.4 \pm 150.1 \\
(413.7-1628.6)\end{array}$ & \\
\hline \multicolumn{5}{|l|}{ Gender } \\
\hline Males (\%) & 59.4 & 58.4 & 61.2 & .865 \\
\hline Females (\%) & 40.6 & 41.6 & 38.8 & .912 \\
\hline Age (years) & $61.9 \pm 6.5^{\circ}$ & $61.9 \pm 6.4^{\circ}$ & $62.5 \pm 6.4$ & .041 \\
\hline BMI $\left(\mathrm{kg} / \mathrm{m}^{2}\right)$ & $30.7 \pm 4.5^{\circ}$ & $30.3 \pm 4.4^{\circ}$ & $29.9 \pm 4.4$ & $<.0001$ \\
\hline Waist Circumference $(\mathrm{cm})$ & $105.5 \pm 11.3$ & $104.3 \pm 11.6$ & $103.6 \pm 10.6$ & .002 \\
\hline Hip circumference $(\mathrm{cm})$ & $107.2 \pm 10.7$ & $106.4 \pm 10.4$ & $105.6 \pm 9.5$ & .005 \\
\hline \multicolumn{5}{|l|}{ Smoking status (\%) } \\
\hline Non smoker & 85.8 & 82.7 & 79.6 & .009 \\
\hline Current smoker & 14.2 & 17.3 & 20.4 & .032 \\
\hline$\%$ on lipid lowering medications & 56.7 & 61.3 & 68.1 & .082 \\
\hline$\%$ on pressure lowering medications & 93.2 & 92.9 & 91.2 & .231 \\
\hline Proteins (\% of TE) & $18.3 \pm 2.4$ & $18.3 \pm 2.4$ & $18.1 \pm 2.7$ & .368 \\
\hline Lipids (\% of TE) & $36.3 \pm 5.9^{\circ}$ & $36.8 \pm 5.6$ & $36.9 \pm 6.4$ & .014 \\
\hline SFA (\% of TE) & $12.4 \pm 2.6^{\circ}$ & $12.3 \pm 2.4^{\circ}$ & $11.8 \pm 2.4$ & $<.0001$ \\
\hline MUFA (\% of TE) & $17.4 \pm 3.5^{\circ}$ & $17.8 \pm 3.4$ & $18.3 \pm 4.1$ & $<.0001$ \\
\hline PUFA (\% of TE) & $4.3 \pm 1.1^{\circ}$ & $4.4 \pm 1.0$ & $4.6 \pm 1.2$ & $<.0001$ \\
\hline Carbohydrates (\% of TE) & $45.4 \pm 7.1$ & $44.9 \pm 6.9$ & $45.1 \pm 7.6$ & .353 \\
\hline Added sugars (\% of TE) & $2.1 \pm 2.9^{\circ}$ & $2.4 \pm 2.8$ & $2.6 \pm 3.7$ & .002 \\
\hline Fiber (g/1000 kcal) & $9.4 \pm 2.1^{\circ}$ & $10.7 \pm 2.4^{\circ}$ & $12.1 \pm 2.9$ & $<.0001$ \\
\hline Alcohol (g/day) & $8.0 \pm 13.9^{\circ}$ & $11.0 \pm 14.4^{\circ}$ & $14.4 \pm 18.5$ & $<.0001$ \\
\hline
\end{tabular}

$\mathrm{M} \pm \mathrm{SD}$.

TE: Total Energy of the diet.

${ }^{\circ} \mathrm{p}<.05$ vs tertile 3, Post-Hoc Test for multiple comparisons (Bonferroni Test).

multiple comparisons). Moreover, a significant linear trend was observed ( $\mathrm{p}<.001$ for all).

The proportion of study participants on lipid lowering agents was equally distributed across the tertiles of polyphenol intake; however, a sensitivity analysis conducted with the exclusion of people on hypolipidemic drugs confirmed all the previous findings (Supplemental Table 1).
Flavonoids and phenolic acids were the two main contributors to the total polyphenol intake in our population; therefore, we analyzed the metabolic variables also according to tertiles of energy-adjusted intake of each of these two classes of polyphenols. The findings were the same as those found with total polyphenol intake (Tables 3 and 4), except for HbA1c, which was no longer significantly related to phenolic acid intake (Table 4).

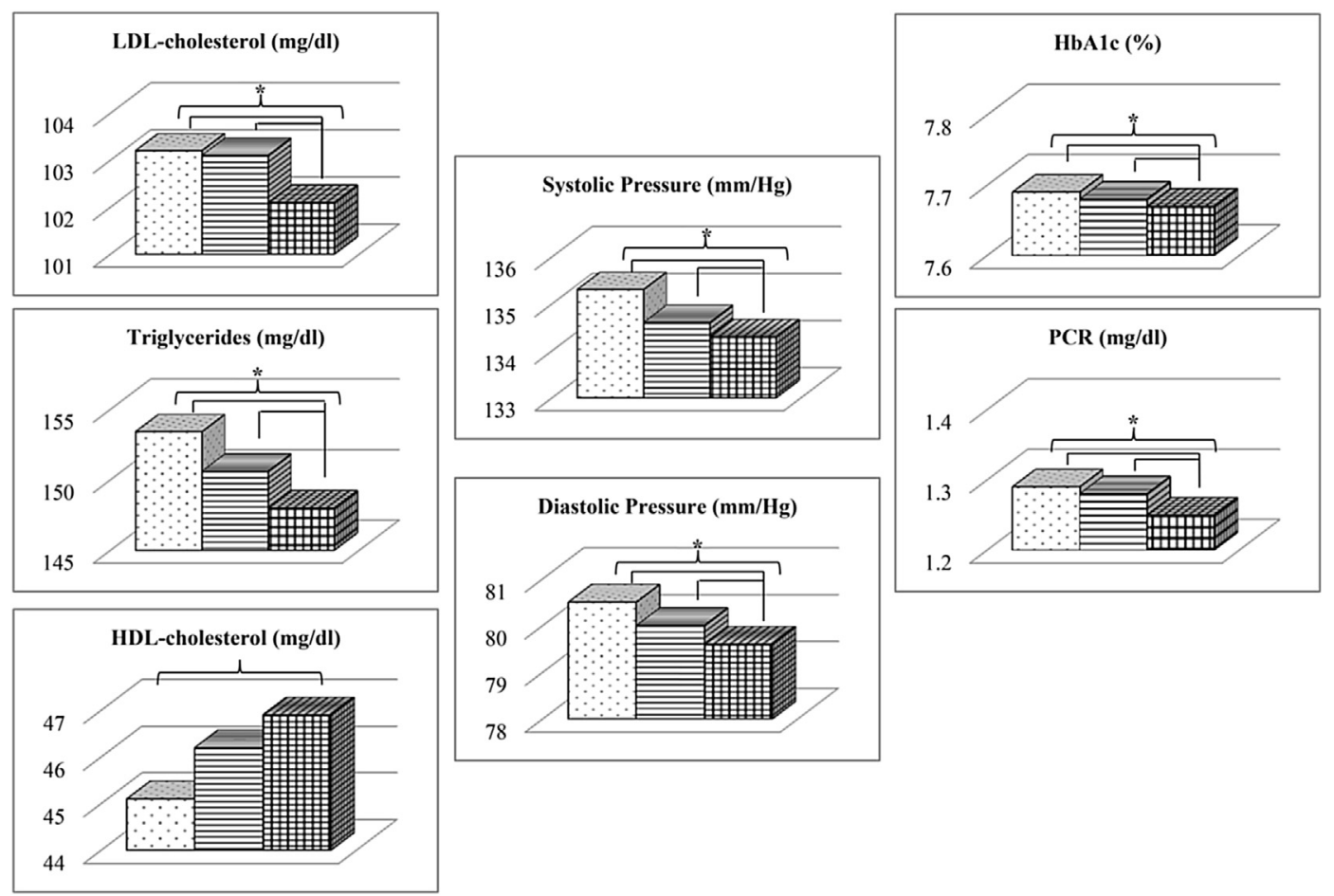

$*_{\mathrm{p}}<.001$, Analysis of Variance and Post-Hoc Test for multiple comparisons (Bonferroni Test) between tertile 3 vs tertiles 1 and 2

Data adjusted for gender, age, BMI, waist and hip circumferences, smoke, alcohol intake, dietary lipids, SFA, MUFA, PUFA, added sugars and fiber

Fig. 1. Metabolic profile, blood pressure values and C-reactive protein according to tertiles of energy-adjusted polyphenol intake ( $\square$ tertile 1 ; $\equiv$ tertile 2 ; $\$$ tertile 3 ). 
Table 3

Metabolic profile, blood pressure values and C-reactive protein according to energy adjusted tertiles of flavonoid intake.

\begin{tabular}{|c|c|c|c|c|}
\hline & Tertile 1 (n 856) & Tertile 2 (n 860) & Tertile 3 (n 858) & P for Anova \\
\hline Flavonoids (mg/1000 Kcal) & $\begin{array}{l}86.1 \pm 23.9 \\
(16.3-121.5)\end{array}$ & $\begin{array}{l}156.2 \pm 21.1 \\
(121.6-193.9)\end{array}$ & $\begin{array}{l}286.7 \pm 118.8 \\
(194.0-1181.3)\end{array}$ & \\
\hline LDL-cholesterol (mg/dl) & $103.3 \pm 3.8^{\circ}$ & $103.0 \pm 3.9^{\circ}$ & $102.1 \pm 3.9$ & $2.2 \times 10^{-8}$ \\
\hline Triglycerides (mg/dl) & $152.9 \pm 18.4^{\circ}$ & $150.7 \pm 16.9^{\circ}$ & $148.3 \pm 17.4$ & $6.5 \times 10^{-8}$ \\
\hline HDL-cholesterol (mg/dl) & $45.2 \pm 4.8^{\circ}$ & $46.2 \pm 4.4^{\circ}$ & $47.0 \pm 4.3$ & $5.3 \times 10^{-15}$ \\
\hline $\operatorname{HbA1c}(\%)$ & $7.69 \pm .09^{\circ}$ & $7.69 \pm .08^{\circ}$ & $7.67 \pm .08$ & $2.1 \times 10^{-7}$ \\
\hline Systolic pressure $(\mathrm{mm} / \mathrm{Hg})$ & $135.2 \pm 3.4^{\circ}$ & $134.5 \pm 3.4$ & $134.6 \pm 3.5$ & $1.9 \times 10^{-4}$ \\
\hline Diastolic pressure $(\mathrm{mm} / \mathrm{Hg})$ & $80.5 \pm 1.7^{\circ}$ & $80.0 \pm 1.7^{\circ}$ & $79.7 \pm 1.8$ & $3.4 \times 10^{-18}$ \\
\hline $\mathrm{CRP}(\mathrm{mg} / \mathrm{dl})$ & $1.29 \pm .15^{\circ}$ & $1.28 \pm .15^{\circ}$ & $1.25 \pm .14$ & $4.3 \times 10^{-10}$ \\
\hline
\end{tabular}

$\mathrm{M} \pm \mathrm{SD}$.

${ }^{\circ} \mathrm{p}<.05$ vs tertile 3, Post-Hoc Test for multiple comparisons (Bonferroni Test).

Data adjusted for gender, age, BMI, waist and hip circumferences, smoke, alcohol intake, dietary lipids, SFA, MUFA, PUFA, added sugars and fiber.

Table 4

Metabolic profile, blood pressure values and C-reactive protein according to energy adjusted tertiles of phenolic acids intake.

\begin{tabular}{|c|c|c|c|c|}
\hline & Tertile $1(856)$ & Tertile 2 (860) & Tertile 3 (858) & P for Anova \\
\hline Phenolic acids (mg/1000 Kcal) & $\begin{array}{l}96.8 \pm 29.3 \\
(18.6-137.5)\end{array}$ & $\begin{array}{l}167.2 \pm 18.2 \\
(137.6-201.5)\end{array}$ & $\begin{array}{l}281.5 \pm 92.0 \\
(201.6-1170.1)\end{array}$ & \\
\hline LDL-cholesterol (mg/dl) & $103.0 \pm 3.8^{\circ}$ & $102.9 \pm 3.9$ & $102.5 \pm 4.1$ & $5.1 \times 10^{-4}$ \\
\hline Triglycerides $(\mathrm{mg} / \mathrm{dl})$ & $152.0 \pm 17.2^{\circ}$ & $150.9 \pm 17.4^{\circ}$ & $148.9 \pm 18.3$ & $7.2 \times 10^{-9}$ \\
\hline HDL-cholesterol (mg/dl) & $45.8 \pm 4.7^{\circ}$ & $46.1 \pm 4.4^{\circ}$ & $46.7 \pm 4.6$ & $1.6 \times 10^{-5}$ \\
\hline $\mathrm{HbA1c}(\%)$ & $7.69 \pm .08$ & $7.68 \pm .08$ & $7.68 \pm .08$ & .217 \\
\hline Systolic pressure (mm/Hg) & $135.5 \pm 3.4^{\circ}$ & $134.8 \pm 3.4^{\circ}$ & $134.0 \pm 3.4$ & $3.1 \times 10^{-19}$ \\
\hline Diastolic pressure $(\mathrm{mm} / \mathrm{Hg})$ & $80.4 \pm 1.7^{\circ}$ & $80.1 \pm 1.6^{\circ}$ & $79.5 \pm 1.8$ & $1.4 \times 10^{-29}$ \\
\hline CRP $(\mathrm{mg} / \mathrm{dl})$ & $1.29 \pm .15^{\circ}$ & $1.27 \pm .15$ & $1.26 \pm .15$ & $1.2 \times 10^{-7}$ \\
\hline
\end{tabular}

$\mathrm{M} \pm \mathrm{SD}$.

${ }^{\circ} \mathrm{p}<.05$ vs tertile 3, Post-Hoc Test for multiple comparisons (Bonferroni Test).

Data adjusted for gender, age, BMI, waist and hip circumferences, smoke, alcohol intake, dietary lipids, SFA, MUFA, PUFA, added sugars and fiber.

\section{Discussion}

This is the first epidemiological observational study evaluating the association between polyphenol intake and major cardiovascular risk factors - glucose control, lipid profile, blood pressure and a biomarker of subclinical inflammation (PCR) in people with type 2 diabetes.

The main and most relevant findings of this study were that a diet characterized by a higher intake of total polyphenols was associated with a better cardiovascular risk factors profile and a lower grade of subclinical inflammation. These findings were independent of other dietary components and potential confounders, such as gender, age, BMI, and smoking; moreover, the associations were evident also in a population at very high cardiovascular risk, who followed a more or less quite homogeneous diet not characterized by a high intake of polyphenols, and who were already on pharmacological treatment for their main cardiovascular risk factors. In addition to the associations with total polyphenol intake, also the consumption of flavonoids and phenolic acids - the two main classes of polyphenols consumed by our population - were significantly associated with a better cardiovascular profile, with the only exception of blood glucose control for phenolic acids. This difference could be explained either by a possible minor influence of phenolic acids on blood glucose metabolism, which should be better investigated in intervention studies, or, more likely, by confounders linked specifically to the intake of phenolic acids. As a matter of fact, the main contributor to phenolic acids intake is represented by non-alcoholic beverages and the added sugars generally utilized in these drinks could have counteracted or masked the relationship between phenolic acids and glucose control. Within this context, it is important to underline that in the same population a positive association was found between added sugars and HbA1c concentrations [28].

Inverse associations between polyphenols, in particular flavonoids, some foods rich in selected polyphenols (fruit and vegetables, coffee, tea, etc.) and cardiovascular risk factors and/or cardiovascular events in the general population have been already reported [11-13]. Our study reinforces, in a different population, the data on flavonoids, extends the results to the other main class of polyphenols - i.e., the phenolic acids - and shows an inverse association also with markers of systemic subclinical inflammation.

Of course, the cross-sectional nature of our study does not allow us to infer a causal effect of polyphenols on the different outcomes. However, intervention studies in humans and in vitro and animal studies support this hypothesis. In fact, intervention trials with single foods/beverages rich in polyphenols (tea, chocolate and cocoa, coffee, wine, orange juice) or extracts of specific polyphenols indicate positive effects on cardiovascular risk factors (plasma lipids, glucose metabolism, blood pressure) and an improvement of the endothelial function $[16,29,30]$. Very recent data show that a diet naturally rich in polyphenols from different sources improves post-prandial lipid metabolism, oxidative stress, and glucose tolerance in metabolically high-risk patients, strongly supporting the possible causal effect of different polyphenols on multiple cardiovascular risk factors $[18,19]$. Moreover, there are also different plausible mechanisms possibly explaining the link between polyphenols and cardiovascular risk factors, which reinforce the possibility of a causal relation. The main one are slow-down in the digestion and absorption of lipids and glucose, increase in insulin secretion and/or decrease in insulin resistance, decrease in oxidative stress and subclinical inflammation, increase in nitric oxide bioavailability with subsequent vasodilatation effect [5,9,31]. Data on the association between high polyphenol intake and lower BMI, waist and hip circumferences, are really interesting and in line with some evidences showing a possible effect of polyphenol in increasing energy expenditure [32]. However, they cannot explain the association between polyphenol and cardiovascular risk factors in our study because the above associations have been found after adjusting for BMI, waist and hip circumferences.

The differences in metabolic profile, blood pressure, and markers of inflammation among the different tertiles of polyphenols and their main classes are statistically significant but their magnitude is 
modest and can hardly be considered of clinical interest, at least at the level of the individual patient; however, it could be relevant when translated at the population level. Moreover, considering the differences between tertiles of polyphenol intake in LDL-cholesterol, triglycerides, HDL-cholesterol and blood pressure, on the basis of prospective and intervention studies [33-37] it is possible to foresee an $11 \%$ reduction in the cardiovascular risk, which is certainly of clinical relevance taking also into account that this reduction is linked to only one component of the diet. Furthermore, from a practical point of view, the main sources of polyphenols in the diet of our population are represented by non-alcoholic beverages, such as tea and coffee (39.5\%), fruit (23.0\%), alcoholic beverages (13.9\%), vegetables (12.4\%), legumes (3.7\%) which, except for alcoholic beverages, represent a good source of nutrients and other bioactive compounds able to positively influence cardiovascular risk factors.

Of course, our study has strengths and limitations.

The large size of the study sample, the selection of a cohort representative of the diabetic population seen in everyday clinical practice, the standardized collection of nutritional data, the centralized measurements of the biochemical variables, and the analysis of different classes of polyphenols, are the major strengths.

Among the limitations, the first one is represented by the crosssectional design of the study, although, as reported previously, intervention and mechanistic studies support a possible causal relation. Other limitations may be linked to the estimation of polyphenol intake: variability or lack of information on polyphenol content in foods depending on ripeness at harvest time, environmental factors, processing and storage, and plant variety; lack of information about some foods in the FFQ, especially for wholegrain foods which could have, if anything, determined an underestimation of the intake of some polyphenols masking some differences.

In conclusion, the results of this study are relevant as they show, for the first time in people with type 2 diabetes, that a diet characterized by a higher intake of polyphenols is associated with a more favorable cardiovascular risk factor profile, and less subclinical inflammation. This is true even if the intake of polyphenols is not particularly high in our population compared to others. This should encourage the use of foods and beverages rich in different classes of polyphenols also in this category of patients as in the general population.

\section{Sources of support}

The study is supported by the Italian Medicines Agency (AIFA) within the Independent Drug Research Program - contract No. FARM6T9CET - and by Diabete Ricerca, the no profit Research Foundation of the Italian Diabetes Society. The funding agency played no role in the study design; in the collection, analysis, and interpretation of data; in the writing of the manuscript; or in the decision to submit the manuscript for publication.

\section{Authors' contributions to the manuscript}

OV, GR, AAR, MM, EB, SDP, CBG, AN, and SS designed research; SA, ACB, RB, EC, MC, MC, GC, SC, LC, FDA, EDA, GDC, LF, GG, SG, CG, RI, CI, AL, DL, LL, CM, SS, LT, RT, and CZ conducted research; LB, SG, and SS provided essential materials; MV, and SG analyzed data and performed statistical analysis; MV, AAR, and GR wrote paper; AAR, MV, and GR had primary responsibility for final content.

All authors read and approved the final manuscript.

\section{Conflict of interest}

All authors disclose any financial or personal relationships with the company or organization sponsoring the research at the time the research was done.

\section{Acknowledgements}

The participation of the patients in the study is gratefully acknowledged. We thank all the investigators and the dietitians in the TOSCA.IT centers for their excellent cooperation.

We thank the "Fondazione Diabete Ricerca", "Società Italiana di Diabetologia" and "MSD Italia s.r.l." for the funding "Borse di Studio - Fondazione Diabete Ricerca - MSD 2015".

\section{Appendix A. Supplementary data}

Supplementary data related to this article can be found at http:// dx.doi.org/10.1016/j.clnu.2016.11.002.

\section{References}

[1] Sies H. Polyphenols and health: update and perspectives. Arch Biochem Biophys 2010;501:2-5.

[2] Gormaz JG, Valls N, Sotomayor C, Turner T, Rodrigo R. Potential role of polyphenols in the prevention of cardiovascular diseases: molecular bases. Curr Med Chem 2016;23(2):115-28.

[3] Rangel-Huerta OD, Pastor-Villaescusa B, Aguilera CM, Gil A. A systematic review of the efficacy of bioactive compounds in cardiovascular disease: phenolic compounds. Nutrients 2015;7(7):5177-216.

[4] Arts IC, Hollman PC. Polyphenols and disease risk in epidemiologic studies Am J Clin Nutr 2005;81(1 Suppl.):317S-25S.

[5] Medina-Remón A, Tresserra-Rimbau A, Pons A, Tur JA, Martorell M, Ros E et al. Effects of total dietary polyphenols on plasma nitric oxide and blood pressure in a high cardiovascular risk cohort. The PREDIMED randomized trial. Nutr Metab Cardiovasc Dis 2015;25(1):60-7.

[6] Guo X, Tresserra-Rimbau A, Estruch R, Martínez-González MA, MedinaRemón A, Castañer O, et al. Effects of polyphenol, measured by a biomarker of total polyphenols in urine, on cardiovascular risk factors after a long-term follow-up in the PREDIMED study. Oxid Med Cell Longev 2016;2016. Article ID 2572606, 11 pages.

[7] Hanhineva K, Törrönen R, Bondia-Pons I, Pekkinen J, Kolehmainen M, Mykkänen $\mathrm{H}$, et al. Impact of dietary polyphenols on carbohydrate metabolism. Int J Mol Sci 2010;11(4):1365-402.

[8] Allen RR, Carson L, Kwik-Uribe C, Evans EM, Erdman Jr JW. Daily consumption of a dark chocolate containing flavanols and added sterol esters affects cardiovascular risk factors in a normotensive population with elevated cholesterol. J Nutr 2008:138:725-31.

[9] Hollenberg NK, Fisher ND, McCullough ML. Flavanols, the Kuna, cocoa consumption, and nitric oxide. J Am Soc Hypertens 2009;3(2):105-12.

[10] Manach C, Scalbert A, Morand C, Rémésy C, Jiménez L. Polyphenols: food sources and bioavailability. Am J Clin Nutr 2004;79(5):727-47.

[11] Grosso G, Stepaniak U, Micek A, Stefler D, Bobak M, Pająk A. Dietary polyphenols are inversely associated with metabolic syndrome in Polish adults of the HAPIEE study. Eur J Nutr 2016 Feb 25. http://dx.doi.org/10.1007/s00394016-1187-z. Epub ahead of print.

[12] Pounis G, Bonaccio M, Di Castelnuovo A, Costanzo S, de Curtis A, Persichillo M, et al. Polyphenol intake is associated with low-grade inflammation, using a novel data analysis from the Moli-sani study. Thromb Haemost 2016;115(2): 344-52.

[13] Zamora-Ros R, Forouhi NG, Sharp SJ, Gonźalez CA, Buijsse B, Guevara M, et al Dietary intakes of individual flavanols and flavonols are inversely associated with incident type 2 diabetes in European populations. J Nutr 2014;144: 335-43.

[14] Tresserra-Rimbau A, Rimm EB, Medina-Remón A, Martínez-González MA López-Sabater MC, Covas MI, et al. Polyphenol intake and mortality risk: a reanalysis of the PREDIMED trial. BMC Med 2014;12:77.

[15] Tresserra-Rimbau A, Guasch-Ferré M, Salas-Salvadó J, Toledo E, Corella D, Castañer O, et al. Intake of total polyphenols and some classes of polyphenols is inversely associated with diabetes in elderly people at high cardiovascular disease risk. J Nutr 2016 Mar 9. http://dx.doi.org/10.3945/jn.115.223610. Epub ahead of print.

[16] Lockyer S, Rowland I, Spencer JP, Yaqoob P, Stonehouse W. Impact of phenolic-rich olive leaf extract on blood pressure, plasma lipids and inflammatory markers: a randomised controlled trial. Eur J Nutr 2016 Mar 7. http:// dx.doi.org/10.1007/s00394-016-1188-y. Epub ahead of print.

[17] Kim Y, Keogh JB, Clifton PM. Polyphenols and glycemic control. Nutrients 2016;8:17. http://dx.doi.org/10.3390/nu8010017. Epub ahead of print.

[18] Bozzetto L, Annuzzi G, Pacini G, Costabile G, Vetrani C, Vitale M, et al. Polyphenol-rich diets improve glucose metabolism in people at high cardiometabolic risk: a controlled randomised intervention trial. Diabetologia 2015;58(7):1551-60.

[19] Annuzzi G, Bozzetto L, Costabile G, Giacco R, Mangione A, Anniballi G, et al. Diets naturally rich in polyphenols improve fasting and postprandial dyslipidemia and reduce oxidative stress: a randomized controlled trial. Am J Clin Nutr 2014;99(3):463-71. 
[20] Grassi D, Lippi C, Necozione S, Desideri G, Ferri C. Short-term administration of dark chocolate is followed by a significant increase in insulin sensitivity and a decrease in blood pressure in healthy persons. Am J Clin Nutr 2005;81:611-4.

[21] Grassi D, Desideri G, Necozione S, Lippi C, Casale R, Properzi G, et al. Blood pressure is reduced and insulin sensitivity increased in glucose-intolerant, hypertensive subjects after 15 days of consuming high-polyphenol dark chocolate. J Nutr 2008;138:1671-6.

[22] Standards of Medical Care in Diabetes 2010. Position statement of the American Diabetes Association (ADA). Diabetes Care 2010:33(Suppl. 1):S11-61.

[23] Vaccaro O, Masulli M, Bonora E, Del Prato S, Giorda CB, Maggioni AP, et al Addition of either pioglitazone or a sulfonylurea in type 2 diabetic patients inadequately controlled with metformin alone: impact on cardiovascular events. A randomized controlled trial. Nutr Metab Cardiovasc Dis 2012;22(11): 997-1006.

[24] Pisani P, Faggiano F, Krogh V, Palli D, Vineis P, Berrino F. Relative validity and reproducibility of a food frequency dietary questionnaire for use in the Italian EPIC centres. Int J Epidemiol 1997;26(Suppl. 1):S152-60.

[25] USDA special interest databases on flavonoids. Nutrient Data Laboratory Home Page: http://www.ars.usda.gov/nutrientdata/flav.

[26] Phenol-Explorer: database on polyphenol content in foods. http://phenolexplorer.eu/.

[27] Parras P, Martínez-Tome M, Jiménez AM, Murcia MA. Antioxidant capacity of coffees of several origins brewed following three different procedures. Food Chem 2007;102:582-92.

[28] Vitale M, Masulli M, Rivellese AA, Babini AC, Boemi M, Bonora E, et al. Influence of dietary fat and carbohydrates proportions on plasma lipids, glucose control and low-grade inflammation in patients with type 2 diabetes-the TOSCA.IT Study. Eur J Nutr 2016;55(4):1645-51.

[29] Hooper L, Kay C, Abdelhamid A, Kroon PA, Cohn JS, Rimm EB, et al. Effects of chocolate, cocoa, and flavan-3-ols on cardiovascular health: a systematic review and meta-analysis of randomized trials. Am J Clin Nutr 2012;95(3):740-51.
[30] Chowdhury A, Sarkar J, Chakraborti T, Pramanik PK, Chakraborti S. Protective role of epigallocatechin-3-gallate in health and disease: a perspective. Biomed Pharmacother 2016;78:50-9.

[31] Desideri G, Kwik-Uribe C, Grassi D, Necozione S, Ghiadoni L, Mastroiacovo D, et al. Benefits in cognitive function, blood pressure, and insulin resistance through cocoa flavanol consumption in elderly subjects with mild cognitive impairment: the Cocoa, Cognition, and Aging (CoCoA) study. Hypertension 2012;60(3):794-801.

[32] Most J, Goossens GH, Jocken JW, Blaak EE. Short-term supplementation with a specific combination of dietary polyphenols increases energy expenditure and alters substrate metabolism in overweight subjects. Int $\mathrm{J}$ Obes (Lond) 2014;38(5):698-706.

[33] Blood Pressure Lowering Treatment Trialists' Collaboration, Turnbull F, Neal B, Ninomiya T, Algert C, Arima H, Barzi F, et al. Effects of different regimens to lower blood pressure on major cardiovascular events in older and younger adults: meta-analysis of randomized trials. BMJ 2008;336:1121-3.

[34] Genser B, Marz W. Low density lipoprotein cholesterol, statins and cardiovascular events: a meta-analysis. Clin Res Cardiol 2006;95(8):393-404.

[35] National Cholesterol Education Program (NCEP) Expert Panel on Detection. Evaluation, and treatment of high blood cholesterol in adults (Adult Treatment Panel III). Third report of the National Cholesterol Education Program (NCEP) expert panel on detection, evaluation, and treatment of high blood cholesterol in adults (Adult Treatment Panel III) final report. Circulation 2002:106(25):3143-421.

[36] Scientific Statement AHA, Miller M, Stone NJ, Ballantyne C, Bittner V, Criqui $\mathrm{MH}$, et al. Triglycerides and cardiovascular disease. Circulation 2011:123:2292-333.

[37] Eeg-Olofsson K, Cederholm J, Nilsson PM, Zethelius B, Svensson AM, Gudbjörnsdóttir S, et al. New aspects of HbA1c as a risk factor for cardiovascular diseases in type 2 diabetes: an observational study from the Swedish National Diabetes Register (NDR). J Intern Med 2010;268(5):471-82. 